$$
\text { Series A }
$$

I. MATHEMATICA

404

\title{
ÜBER QUASIRATIONALE FUNKTIONEN AUF PARABOLISCHEN RIEMANNSCHEN FLÄCHEN \\ VON
}

LAURI MYRBERG

H E L S I N K I 1967

S U O M A L A INEN TIEDEAKATEMIA

doi:10.5186/aasfm.1967.404 
Am 10 Februar 1969 vorgelegt von OLLi Lehto und K. I. Virtanen. 


\section{Über quasirationale Funktionen auf parabolischen Riemannschen Flächen}

Eine auf der Riemannschen Fläche $F$ definierte komplexwertige Funktion $f$ heisst meromorph, wenn sie auf $F$ bis auf Pole analytisch ist. Wenn $F$ kompakt ist, muss die Anzahl der Pole endlich sein, und $f$ ist eine rationale Funktion, die alle Werte der erweiterten komplexen Ebene genau $n$-mal annimmt, wo $n$ endlich ist. Eine andere wichtige Eigenschaft von rationalen Funktionen ist die Körpereigenschaft. Versucht man auf einer nichtkompakten Fläche eine ähnliche Funktionenklasse zu definieren, so ist es notwendig zu fordern, dass die Funktionen auf dem idealen Rande sich genügend regulär verhalten. Ist die Riemannsche Fläche parabolisch, erweist es sich als zweckmässig zu fordern, dass diese "quasirationalen» Funktionen in jedem Punkt des idealen Randes einen (endlichen oder unendlichen) Grenzwert besitzen. Dadurch erhalten wir eine Menge von meromorphen Funktionen, die u.a. eine endliche Valenz und die Körpereigenschaft haben. Sie haben auch einen engen Zusammenhang mit meromorphen Differentialen, die ausserhalb einer kompakten Menge quadratisch integrierbar sind.

Einen Teil von den in dieser Arbeit vorkommenden Hilfssätzen hat M. Heins [3] in dem Fall bewiesen, dass die Riemannsche Fläche nur eine Randkomponente besitzt. Ein Teil unserer Resultate gilt allgemeiner, als wir hier formuliert haben (Royden [10], [11], Ozawa [9]). Das vorliegende Problem ist auch von Ahlfors [1], Tsusi [12] und vom Verf. [4], [5],[6],[7], behandelt worden.

\section{Die Randkomponenten einer Riemannschen Fläche}

1. Es sei $F$ eine nichtkompakte Riemannsche Fläche. Unter einer Randkomponente von $F$ versteht man eine nichtleere Familie $R$ von Teilgebieten $Q$ von $F$, welche die folgenden Eigenschaften besitzt ([2] S. 82):

$1^{\circ}$ Gehört $Q$ zu $R$, so ist $\bar{Q}$ nichtkompakt.

$2^{\circ}$ Der Rand $\partial Q$ von $Q \in R$ ist kompakt.

$3^{\circ}$ Aus $Q_{0} \in R$ und $Q \supset Q_{0}$ folgt $Q \in R$. 
$4^{\circ}$ Wenn $Q_{1}$ und $Q_{2}$ zu $R$ gehören, so gibt es ein Gebiet $Q \in R$ so dass $Q \subset Q_{1} \cap Q_{2}$.

$5^{\circ}$ Der Durchschnitt $\bigcap_{Q \in R} \bar{Q}$ ist leer.

Die Menge? aller Randkomponenten von $F$ heisst der ideale Rand von $F$. Ist $Q$ ein gegebenes Gebiet, so bedeutet $g(Q)$ die Menge aller Randkomponenten $R$, für welche $Q \in R$ gilt.

Die Riemannsche Fläche $F$ kann zu einem kompakten topologischen Raum $M=F \cup$, komplettiert werden, indem man in $M$ als Basis von offenen Mengen die folgenden Mengen wählt:

$1^{\circ}$ Alle offenen Mengen von $F$.

$2^{\circ}$ Alle Mengen von der Form $Q \cup 9(Q)$, wo $Q$ zu einer Randkomponente gehört.

Der so entstandene topologische Raum ist ein kompakter HausdorffRaum, der die Fläche $F$ als eine dichte Teilmenge enthält.

\section{Lokale Eigenschaften von meromorphen Funktionen auf parabolischen Riemannschen Flächen}

2. Es sei $Q$ ein nichtkompaktes Gebiet von $F$, dessen Rand $\partial Q$ aus einer endlichen Menge von geschlossenen analytischen Kurven besteht, und $\Im(Q)$ der zugehörige Teil des idealen Randes. Eine Funktion $f$ ist in $\bar{Q}$ meromorph, wenn sie in $\bar{Q}$ bis auf Pole analytisch ist. Die Pole von $f$ können sich somit höchstens auf $Y(Q)$ häufen. Ist $w$ ein Punkt der erweiterten komplexen Ebene $\bar{C}$, bezeichnen wir mit $n(f, w, Q)$ die Anzahl der $w$-Stellen von $f$ in $Q$, wobei die $k$-fachen $w$-Stellen $k$-mal berechnet werden.

Wir werden im folgenden nur parabolische Riemannsche Flächen betrachten. Eine Riemannsche Fläche $F$ ist parabolisch, wenn auf $F$ keine nichtkonstanten negativen subharmonischen Funktionen existieren. Auf $F$ ist dann das erweiterte Maximumprinzip der harmonjschen Funktionen gültig.

3. Es sei also $F$ eine parabolische Riemannsche Fläche und $Q$ ein nichtkompaktes Teilgebiet von $F$, dessen Rand aus einer endlichen Menge von geschlossenen analytischen Kurven besteht. Es sei ferner $f$ eine nichtkonstante in $Q$ beschränkte analytische Funktion: $f(z)<1$. Wir werden das Verhalten von $f$ in der Nähe des idealen Randes 'M $(Q)$ untersuchen.

Hilfssatz 2.1. Die Funktion $n(f, w, Q)$ ist beschränkt:

$$
n(f, w, Q) \leqq N
$$

für alle $w \in C$. 
Beweis. Es sei $g(z, x)$ die Greensche Funktion von $Q$ mit dem Pol in $x$ und $w$ eine komplexe Zahl, so dass $|w| \leqq 1$. Dann gilt wegen des erweiterten Maximumprinzips in $Q$ die Ungleichung

$$
\sum_{f\left(x_{n}\right)=w} g\left(z, x_{n}\right) \leqq \log \left|\frac{1-\bar{w} f(z)}{f(z)-w}\right| .
$$

Es sei nun $w_{0}$ eine komplexe Zahl, so dass $\left|w_{0}\right| \leqq 1, U\left(w_{0}, r\right)$ eine Kreisumgebung von $w_{0}$ und $z_{0}$ ein fester Punkt von $Q$, so dass $f\left(z_{0}\right)$ nicht zu $\overline{U\left(w_{0}, r\right)}$ gehört. Dann folgt aus (2.1) für alle Punkte $w \in U\left(w_{0}, r\right)$ die Ungleichung

$$
\sum_{f\left(x_{n}\right)=w} g\left(z_{0}, x_{n}\right) \leqq \operatorname{Max}_{w \in \overline{U\left(w_{0}, r\right)}} \log \left|\frac{1-\bar{w} f\left(z_{0}\right)}{f\left(z_{0}\right)-w}\right| .
$$

Nun ist wegen des erweiterten Maximumprinzips

$$
\inf _{x \in Q^{*}} g\left(z_{0}, x\right)>0,
$$

wo $\bar{Q}^{*} \subset Q$ und $\bar{Q}-Q^{*}$ kompakt ist. Dann folgt aus (2.2), dass die Anzahl der $w$-Stellen von $f$ in $Q$ für $w \in \overline{U\left(w_{0}, r\right)}$ beschränkt ist. Weil die Umgebungen $U\left(w_{0}, r\right)$ eine offene Überdeckung der kompakten Menge $\overline{U(0,1)}$ bilden, folgt hieraus die Behauptung des Hilfssatzes.

Hilfssatz 2.2. Die Funktion $f$ hat einen Grenzwert in jeder Randkomponente $R$ von $g(Q)$.

Beweis. Es sej $R$ eine Randkomponente von $g(Q)$ und $\left\{Q_{n}\right\}$, $n=1,2, \ldots$ eine Folge von Teilgebieten von $Q$, so dass $Q_{n} \in R, \bar{Q}_{n+1} \subset \bar{Q}_{n}$ und $\bigcap_{n=1}^{\infty} Q_{n}=\varnothing$. Dann ist die Menge $K=\bigcap_{n=1}^{\infty} \overline{f\left(Q_{n}\right)}$ abgeschlossen und zusammenhängend. Es genügt zu zeigen, dass sie nur einen Punkt enthält. Antithese: Die Menge $K$ ist ein Kontinuum. Weil die Funktion $n(f, w, Q)$ beschränkt ist, gibt es einen Punkt $w_{0} \in K$, wo $n(f, w, Q)$ den Maximumwert Max $n(f, w, Q)$ erreicht. Hieraus folgt, dass es ein Kontinuum $K_{0} \subset K$ und eine ganze Zahl $m$ gibt, so dass $w_{0} \in K_{0}$ und $f\left(\bar{Q}_{m}\right) \cap K_{0}=\varnothing$. Wir können $K_{0}$ so wählen, dass das Komplement $G=\bar{C}-K_{0}$ zusammenhängend ist und den Punkt $w=\infty$ enthält. Es sei $g(w, \infty)$ die Greensche Funktion von $G$ mit dem $\mathrm{Pol}$ in $\infty$. Dann ist die zusammengesetzte Funktion $g(f(z), \infty)$ in $\bar{Q}_{m}$ harmonisch und positiv, und

$$
\inf _{z \in \bar{Q}_{m}} g(f(z), \infty)=0,
$$

weil $g(w, \infty)$ auf $K_{0}$ den Grenzwert Null hat und $K_{0} \subset \overline{f\left(Q_{m}\right)}$ ist. Dies steht mit dem erweiterten Maximumprinzip im Widerspruch, woraus folgt, dass die Antithese falsch ist. 
Trivialerweise gilt auch das folgende umgekehrte Resultat:

Hilfssatz 2.3. Hat eine in $Q$ analytische Funktion $f$ einen endlichen Grenzwert in einer Randkomponente $R$ von $Q$, so ist $f$ in einem Gebiet $Q_{0} \in R$ beschränkt.

Die Hilfssätze 2.1 und 2.2 sind auch in der folgenden modifizierten Form gültig:

Hilfssatz 2.1.a. Ist die Funktion $f$ in $\bar{Q}$ meromorph, und gibt es eine komplexe Zahl a und eine positive reelle Zahl $M$ so dass in $Q$ die Ungleichung

$$
|f(z)-a|^{-1}<M \text { bzw. }|f(z)|<M
$$

gilt, so ist die Funktion $n(f, w, Q)$ in $\bar{C}$ beschränkt.

Hilfssatz 2.2.a. Unter derselben Bedingung wie im Hilfssatz 2.1.a. besitzt die Funktion $f$ einen (endlichen oder unendlichen) Grenzwert in jeder Randkomponente von $Q$.

4. Es sei $f$ meromorph in einer Umgebung $Q_{0}$ einer Randkomponente $R$ (d.h. in einem Gebiet $Q_{0} \in R$ ). Betreffend das Verhalten von $f$ in der Nähe von $R$ gibt es zwei wesentlich verschiedene Fälle:

$1^{\circ}$ Für eine Umgebung $Q$ von $R$ ist die Menge $\overline{f(Q)}$ eine echte Teilmenge von $\bar{C}$.

Es ist dann leicht einzusehen, dass für passende $a$ und $M$ die Bedingung (2.3) erfüllt ist, woraus folgt, dass $f$ in $R$ einen Grenzwert besitzt. $2^{\circ}$ Für alle Umgebungen $Q$ von $R$ stimmt die Menge $\overline{f(Q)}$ mit $\bar{C}$ überein.

In diesem Fall gilt das folgende Resultat:

Die Funktion $f$ nimmt alle Werte von $\bar{C}$, höchstens von einer Menge von Kapazität Null abgesehen (kürzer: bis auf Nullmenge), in jeder Umgebung von $R$ unendlich oft an.

Beweis. Es sei $\left\{Q_{n}\right\}, n=1,2, \ldots$ eine Folge von Mengen, so dass $Q_{n} \in R, \bar{Q}_{n+1} \subset \bar{Q}_{n}$ für alle $n$ und $\bigcap_{n=1}^{\infty} \bar{Q}_{n}=\varnothing$. Die Mengen

$$
E_{n}=\left\{w \in \bar{C} \mid n\left(f, w, Q_{n}\right)=0\right\}
$$

sind abgeschlossen, $E_{n+1} \supset E_{n}$ für alle $n$, und

$$
\left\{w \in \bar{C} \mid n\left(f, w, Q_{0}\right)<\infty\right\}=\bigcup_{n=1}^{\infty} E_{n} .
$$

Es genügt zu zeigen, dass die Kapazität von $E_{n}$ für alle $n$ gleich Null ist. Antithese: Kap $E_{m}>0$ für ein $m$. Dann gibt es eine Teilmenge $E_{m}^{\prime} \subset E_{m}$ mit folgenden Eigenschaften: 1) $E_{m}^{\prime}$ ist abgeschlossen, 2) Das Komplement 
$\bar{C}-E_{m}^{\prime}$ ist zusammenhängend, 3) Die Kapazität von $E_{m}^{\prime}$ ist positiv. Wegen 3) existiert in $\bar{C}-E_{m}^{\prime}$ eine harmonische Funktion $u$ mit

$$
u\left(\bar{C}-E_{m}^{\prime}\right)=(0,1) .
$$

Dann ist die Funktion $u^{*}=u \circ f$ in $Q_{m}$ harmonisch mit $u^{*}\left(Q_{m}\right)=(0,1)$. Weil aber $\overline{f\left(Q_{n}\right)}=\bar{C}$ für alle $n$ ist, gilt $u^{*}\left(Q_{n}\right)=(0,1)$ für alle $n \geqq m$. Andererseits ist $u^{*}<1$ auf $\partial Q_{m+1}$, was mit dem erweiterten Maximumprinzip im Widerspruch steht. Damit ist die Behauptung bewiesen. Zusammenfassend haben wir den folgenden

Satz 2.1. Es sei $f$ meromorph in einer Umgebung $Q_{0}$ einer Randkomponente $R$. Dann ist entweder

$1^{\circ}$ für eine Umgebung $Q$ von $R$ die Menge $\overline{f(Q)}$ eine echte Teilmenge von $\bar{C}$, oder

$2^{\circ} \overline{f(Q)}=\bar{C}$ für alle $Q \in R$.

Im ersten Fall hat $f$ in $R$ einen Grenzwert, im zweiten gilt für alle $Q \in R$ und für alle $w \in \bar{C}$ (bis auf Nullmenge) die Gleichung $n(f, w, Q)=\infty$.

5. Mit Hilfe des vorigen Satzes erhalten wir das folgende Resultat betreffend das Verhalten des Quotienten zweier beschränkten analytischen Funktionen:

Hilfssatz 2.4. Haben zwei beschränkte analytische Funktionen $f$ und $g$ (die nicht identisch verschwinden) in einer Randkomponente $R$ einen Grenzwert, so besitzt auch der Quotient $f / g$ in $R$ einen (endlichen oder unendlichen) Grenzwert.

Beweis. Es genügt, den Fall zu betrachten, dass

$$
\lim _{z \rightarrow R} f(z)=\lim _{z \rightarrow R} g(z)=0 .
$$

Es sei $Q$ eine Umgebung von $R$, so dass $f, g \neq 0$ in $\bar{Q}$. Dann ist

$$
[n(f / g, w, Q)=n(f-w g, 0, Q)<\infty
$$

für alle $w \in C$. Nach dem Satz 2.1 hat die Funktion $f / g$ einen Grenzwert in $R$.

Aus dem obigen Hilfssatz folgt unmittelbar das folgende allgemeinere Resultat:

Hilfssatz 2.4.a. Sind $f$ und $g$ zwei nicht identisch verschwindende meromorphe Funktionen, die in einer Randkomponente $R$ Grenzwerte besitzen, so hat auch der Quotient $f / g$ in $R$ einen Grenzwert.

6. Mit Rücksicht auf den Satz 2.1 ist es natürlich, die hebbare Singularität, den Pol und die wesentliche Singularität einer meromorphen Funktion in einer Randkomponente auf folgende Weise zu definieren: 
Definition 2.1. Die Randkomponente $R$ ist für eine meromorphe Funktion $f$

a) eine hebbare Singularität (im erweiterten Sinne), wenn $\lim _{z \rightarrow R} f(z)$ existiert und endlich ist,

b) ein Pol (im erweiterten Sinne), wenn $\lim _{z \rightarrow R} f(z)=\infty$ ist,

c) eine wesentliche Singularität (im erweiterten Sinne), wenn $\lim _{z \rightarrow R} f(z)$ nicht existiert.

Hat die Funktion $f$ in einer Randkomponente $R$ einen endlichen Grenzwert $w$, so setzen wir $f(R)=w$. Ist der Grenzwert unendlich, so setzen wir $f(R)=\infty$. Ist die Funktion $f$ im Gebiet $Q_{0}$ meromorph, definieren wir den Wert $f(R)$ in allen Randkomponenten $R \in \mathcal{g}\left(Q_{0}\right)$, wo es auf die obige Weise möglich ist. Es gilt dann der

Hilfssatz 2.5. Die fortgesetzte Funktion ist stetig (in der Topologie von $M=F \cup 9$ bzw. von $\bar{C})$ in allen Punkten von $g\left(Q_{0}\right)$, wo sie definiert ist.

Beweis. Es sei $R_{0} \in \mathcal{Y}\left(Q_{0}\right)$ ein Punkt, wo $f\left(R_{0}\right)$ endlich ist. Die Umgebungen von $R_{0}$ in $M$ haben eine Basis von der Form $\{Q \cup 9(Q)\}$, wo $Q \in R_{0}$. Weil $\lim f(z)=f\left(R_{0}\right)$ ist, gibt es für jedes $\varepsilon>0$ eine Menge $\underset{\substack{z \in F \\ z \rightarrow R_{0}}}{(z)}$

$Q_{\varepsilon} \in R_{0}$ so dass $\left|f(z)-f\left(R_{0}\right)\right|<\varepsilon$ für jedes $z$ in $Q_{\varepsilon}$ gilt. Nach dem Hilfssatz 2.2 hat die Funktion $f$ einen endlichen Grenzwert in jedem Punkt $R \in 9\left(Q_{\varepsilon}\right)$, und es gilt $\left|f(R)-f\left(R_{0}\right)\right| \leqq \varepsilon$. Somit ist die Ungleichung $\left|f(z)-f\left(R_{0}\right)\right| \leqq \varepsilon$ in allen Punkten $z$ von $Q_{\varepsilon} \cup 9\left(Q_{\varepsilon}\right)$ gültig, und die Funktion $f$ ist in $R_{0}$ stetig. In derselben Weise kann der Fall $f\left(R_{0}\right)=\infty$ behandelt werden.

7. Wir definieren noch die Multiplizität des Funktionwertes $f(R)$ in einer Randkomponente $R$. Ist für ein $Q \in R$

$$
N(f, Q)=\operatorname{Max}_{w \in \bar{c}} n(f, w, Q),
$$

definieren wir die Multiplizität $n(f, f(R), R)$ von $f(R)$ durch die Formel

$$
n(f, f(R), R)=\operatorname{Min}_{Q \in R} N^{N}(f, Q) .
$$

Aus der Definition folgt sofort: Es gibt ein $Q_{0} \in R$, so dass die Gleichung

$$
n(f, f(R), R)=N(f, Q)
$$

für alle $Q \in R$ mit $Q \subset Q_{0}$ gültig ist.

8. Im Hilfssatz 2.1 wurde bewiesen, dass für eine beschränkte Funktion $f$ die Anzahl $n(f, w, Q)$ der $w$-Stellen beschränkt ist. Wir wollen nun genauer untersuchen, wie oft eine Funktion von beschränkter Valenz 
wirklich einen gegebenen Wert $w \in \bar{C}$ annimmt. Wir beweisen zuerst den folgenden

Hilfssatz 2.6. Es seien ein Gebiet $Q$ der Fläche $F$ und eine Funktion $f$ gegeben, so dass die folgenden Bedingungen erfüllt sind:

$1^{\circ}$ Die Menge $\bar{Q}$ ist nichtkompakt.

$2^{\circ}$ Der Rand $\partial Q$ von $Q$ besteht aus einer endlichen Menge von geschlossenen analytischen Kurven.

$3^{\circ}$ Die Funktion $f$ ist in $\bar{Q}$ meromorph.

Es seien ferner

$4^{\circ} K$ eine Komponente des Komplements von $f(\partial Q)$.

$5^{\circ} Q_{K}$ eine Komponente von $f^{-1}(K)$.

Dann überdeckt die Komponente $Q_{K}$ alle Punkte von $K$ (bis auf Nullmenge $E)$, d.h. $n\left(f, w, Q_{K}\right)>0$ für $w \in K-E$.

Beweis. Antithese: Die Kapazität der Ausnahmemenge ist positiv. Dann gibt es eine Teilmenge $E^{\prime}$ von $E$ mit folgenden Eigenschaften: 1) $E^{\prime}$ ist abgeschlossen. 2) Das Komplement $K-E^{\prime}$ ist zusammenhängend. 3) Die Kapazität von $E^{\prime}$ ist positiv. Wegen 3) existiert in $K-E^{\prime}$ eine harmonische Funktion $u$ mit $\lim _{w \rightarrow \partial K} u(w)=0$ und $\sup _{K-E^{\prime}} u=1$. Dann jst die Funktion $u^{*}=u \circ f$ in $Q_{K}$ nichtkonstant, harmonisch und beschränkt, und $\lim _{z \rightarrow \partial Q_{K}} u^{*}(z)=0$. Dies ist aber unmöglich, weil $Q_{K}$ ein Gebiet einer parabolischen Fläche ist. Die Antithese ist somit falsch.

Mit Hilfe des vorigen Hilfssatzes kann das folgende schärfere Resultat erhalten werden:

Hilfssatz 2.7. Es sei angenommen:

$1^{\circ}-4^{\circ}$ wie im Hilfssatz 2.6 .

$5^{\circ} Q_{K}$ ist eine Komponente von $f^{-1}(K)$, so dass $\operatorname{Max} n\left(f, w, Q_{K}\right)=n<\infty$. $w \in K$

Dann überdeckt die Komponente $Q_{K}$ (bis auf Nullmenge $E_{n}$ ) alle Punkte von $K n$-mal.

Beweis. Es sei

$$
E_{k}=\left\{w \in K \mid n\left(f, w, Q_{K}\right)<k\right\}, k=1,2, \ldots
$$

Es gilt $E_{1} \subset E_{2} \subset \ldots, E_{k}=K$ für $k>n$, und die Kapazität von $E_{1}$ ist (nach dem Hilfssatz 2.6) gleich Null. Es genügt zu zeigen, dass die Kapazität der Menge

$$
D_{k}=E_{k+1}-E_{k}=\left\{w \in K \mid n\left(f, w, Q_{K}\right)=k\right\}
$$

für $k=1, \ldots, n-1$ gleich Null ist. Antithese: Für ein $m(1 \leqq m \leqq n-1)$ ist die Kapazität von $D_{m}$ positiv, von $E_{m}$ gleich Null. Dann gibt es eine Menge $D_{m}^{\prime} \subset D_{m}$ und eine Kreisumgebung $U\left(w_{0}, r\right) \subset K$ mit folgenden Eigenschaften: 1) $w_{0} \in D_{m}^{\prime}$, 2) $D_{m}^{\prime}$ ist abgeschlossen, 3) $D_{m}^{\prime} \subset$ 
$\left.U\left(w_{0}, r\right), 4\right)$ Die Kapazität von $D_{m}^{\prime}$ ist positiv, 5) Das Komplement $K-D_{m}^{\prime}$ ist zusammenhängend, 6) Es gibt eine endliche Anzahl $s(\leqq m)$ von Komponenten $Q_{U}^{i}$ von $f^{-1}\left(U\left(w_{0}, r\right)\right)$, so dass a) $Q_{U}^{i}$ kompakt ist und der Rand $\partial Q_{U}^{i}$ aus einer endlichen Menge von geschlossenen analytischen Kurven besteht und b) die Menge $\bigcup_{i=1}^{s} Q_{U}^{i}$ den Kreis $U\left(w_{0}, r\right)$ genau $m$-mal überdeckt, 7) $\operatorname{Max} n\left(f, w, Q_{K}\right)>m$. Wegen 7) gibt es noch wenigstens eine Komponente $Q_{U}^{s+1}$ von $f^{-1}\left(U\left(w_{0}, r\right)\right)$; andererseits sind die Bedingungen des Hilfssatzes 2.6 erfüllt, wenn man statt der Mengen $Q, K$ und $Q_{K}$ die Mengen $Q-\bigcup_{i=1}^{s} \bar{Q}_{U}^{i}, U\left(w_{0}, r\right)$ und $Q_{U}^{s+1}$ betrachtet. Nach dem erwähnten Hilfssatz überdeckt $Q_{U}^{s+1}$ alle Punkte von $U\left(w_{0}, r\right)$ (bis auf Nullmenge). Nun überdeckt aber $Q_{U}^{s+1}$ die Menge $D_{m}^{\prime}$ nicht, was wegen 4) zu einem Widerspruch führt. Die Antithese ist somit falsch, und der Hilfssatz ist bewiesen.

\section{3. Über quasirationale Funktionen}

9. Nach diesen lokalen Betrachtungen gehen wir zu den globalen Eigenschaften von meromorphen Funktionen über.

Definition 3.1. Eine auf einer parabolischen Riemannschen Fläche $F$ meromorphe Funktion $f$ heisst quasirational (kurz $Q R$ ), wenn sie in $M=$ FU9 keine andere Singularitäten als Pole besitzt, also speziell in einer Randkomponente höchstens einen Pol (im erweiterten Sinne) hat.

Es kann geschehen, dass auf einer gegebenen Riemannschen Fläche nur konstante $Q R$-Funktionen existieren. Wir nehmen im folgenden an, dass dieser triviale Fall nicht vorliegt, und beweisen einen Satz, der die wichtigsten Eigenschaften einer $Q R$-Funktion enthält.

Satz 3.1. Es sei $f$ eine auf einer parabolischen Riemannschen Fläche $F$ nichtkonstante QR-Funktion. Dann gilt:

$1^{\circ} f$ hat in jeder Randkomponente einen Grenzuert.

$2^{\circ} f$ hat eine beschränkte Valenz: $n(f, u, F) \leqq \Lambda<\infty$ für alle $w \in \bar{C}$. $3^{\circ} n(f, w, F)=N=\operatorname{Max} n(f, w, F)$ für alle $w \in \bar{C}-E$, wo $E$ eine

Nullmenge ist.

$$
w \in \bar{C}
$$

$4^{\circ} f$ kann in der Form

$$
f=a+\frac{1}{f_{1}}
$$

dargestellt werden, wo $f_{1}$ ausserhalb einer kompakten Menge beschränkt ist. 
Beweis. $1^{\circ}$ Die Behauptung enthält nur die Definition einer nichtwesentlichen Singularität.

$2^{\circ}$ Weil $f$ einen (endlichen oder unendlichen) Grenzwert in allen Randkomponenten besitzt, hat jeder Punkt $z$ von $M$ eine Umgebung $Q(z)$ $\cup \mathscr{S}(Q(z))$, so dass $f$ bzw. $1 / f$ in $Q(z)$ beschränkt ist. Nach dem Hilfssatz 2.1.a ist dann die Funktion $n(f, w, Q(z))$ in $\bar{C}$ beschränkt:

$$
n(f, w, Q(z)) \leqq n(z)
$$

für alle $w \in \bar{C}$. Die Behauptung folgt dann aus der Kompaktheit von $M$.

$3^{\circ}$ Es sei $U\left(w_{0}, r\right)$ eine Kreisumgebung der $w$-Ebene, so dass $n(f, w, F)$ $=N=\operatorname{Max} n(f, w, F)$ für alle $w$ in $U\left(w_{0}, r\right)$ gilt, die Menge $\overline{f^{-1}\left(U\left(w_{0}, r\right)\right)}$ $w \in \bar{C}$

kompakt und ihr Komplement zusammenhängend ist. Dann hat die Kreisumgebung $U\left(w_{0}, \frac{r}{2}\right)$ die folgenden Eigenschaften: 1) Die Menge $\overline{f^{-1}\left(U\left(w_{0}, \frac{r}{2}\right)\right)}$ ist kompakt. 2) Die Menge $f^{-1}\left(\partial U\left(w_{0}, \frac{r}{2}\right)\right)$ besteht aus einer endlichen Menge von geschlossenen analytischen Kurven. 3) Das Komplement von $\partial U\left(w_{0}, \frac{r}{2}\right)$ hat zwei Komponenten, $K_{1}=U\left(w_{0}, \frac{r}{2}\right)$ und $K_{2}$, und $\operatorname{Max}_{w \in K_{i}} n(f, w, F)=N$ für $i=1,2$. Dann ist also für $Q_{K_{2}}=f^{-1}\left(K_{2}\right)$ $\operatorname{Max} n\left(f, w, Q_{K_{2}}\right)=N$. Aus dem Hilfssatz 2.7 folgt (wenn man $K=K_{2}$ und $Q_{K}=Q_{K_{2}}$ wählt), dass die Menge $Q_{K_{2}}$ die Menge $K_{2}$ bis auf Nullmenge $N$-mal überdeckt. Auch die Menge $\bar{K}_{1}$ ist von $F N$-mal überdeckt, und die Behauptung ist bewiesen.

$4^{\circ}$ Es sei $w_{0}$ ein Punkt, so dass $n\left(f, w_{0}, F\right)=N$ ist. Dann gibt es eine Umgebung $U\left(w_{0}, r\right)$, so dass die Menge $\overline{f^{-1}\left(U\left(w_{0}, r\right)\right)}$ kompakt ist. Ausserhalb dieser Menge ist $\left|f(z)-w_{0}\right|>r$ und $\left|f(z)-w_{0}\right|^{-1}<\frac{1}{r}$. Bezeichnet man

$$
f_{1}(z)=\left(f(z)-w_{0}\right)^{-1}
$$

woraus

$$
f(z)=w_{0}+\frac{1}{f_{1}(z)}
$$
folgt, so ist $\left|f_{1}(z)\right|<\frac{1}{r}$ ausserhalb von $\overline{f^{-1}\left(U\left(w_{0}, r\right)\right)}$, und die Behauptung
ist bewiesen.

Bemerkung. Nimmt die Funktion $f$ den Wert $\infty$ genau $N$-mal an, so ist $f$ ausserhalb einer kompakten Menge beschränkt. 
Wenn eine auf $F$ meromorphe Funktion $f$ nicht $Q R$ ist, so gibt es wenigstens eine Randkomponente $R$, wo $f$ keinen Grenzwert besitzt. Nach dem Satz 2.1 stimmt dann die Menge $\overline{f(Q)}$ für alle $Q \in R$ mit $\bar{C}$ überein, und $n(f, w, Q)=\infty$ für alle $w$ (bis auf Nullmenge). Wir haben somit den folgenden

Satz 3.2. Ist die meromorphe Funktion $f$ nicht quasirational, so nimmt $f$ alle Werte $w$ von $\bar{C}$ (bis auf Nullmenge) unendlich oft an.

10. Wir geben nun einige Bedingungen, unter welchen eine meromorphe Funktion quasirational ist.

Satz 3.3. Eine auf $F$ meromorphe Funktion $f$ ist quasirational, wenn eine der folgenden Bedingungen erfüllt ist:

$1^{\circ} f$ ist beschränkt ausserhalb einer kompakten Menge.

$2^{\circ}$ Das Dirichletintegral

$$
D_{F-K}(f)=\int_{F-K} \int_{K}\left|f^{\prime}(z)\right|^{2} d \sigma \quad(K \text { kompakt })
$$

ist endlich.

$3^{\circ}$ Der sphärische Inhalt

$$
A_{F}(f)=\iint_{F} \frac{\left|f^{\prime}(z)\right|^{2}}{\left(1+|f(z)|^{2}\right)^{2}} d \sigma
$$

derjenigen Riemannschen Fläche, auf welche die Funktion $f$ die Fläche $F$ abbildet, ist endlich.

$4^{\circ}$ Die Funktion $n(f, w, F)$ ist endlich in einer Menge $E \subset \bar{C}$, deren Kapazität positiv ist.

Beweis. $1^{\circ}$ Nach dem Hilfssatz 2.2 hat $f$ einen endlichen Grenzwert in allen Randkomponenten von $F$ und ist somit $Q R$.

$2^{\circ}$ Auf einer parabolischen Riemannschen Fläche impliziert die Bedingung $2^{\circ}$ die Bedingung $1^{\circ}$.

$3^{\circ}$ Antithese: $f$ ist nicht $Q R$. Dann nimmt $f$ (bis auf Nullmenge) alle Werte $w$ mit $|w|<1$ unendlich oft an, woraus folgt, dass das über die Menge $F_{1}=\{z \in F|| f(z) \mid<1\}$ genommene Dirichletintegral unendlich ist. Wegen

$$
A_{F_{1}}(f)>\frac{1}{4} D_{F_{1}}(f)
$$

gilt dasselbe auch für den sphärischen Inhalt, was einen Widerspruch enthält.

$4^{\circ}$ Die Behauptung folgt aus dem Satz 3.2. 
11. Nach dem Satz 3.1 nimmt eine nichtkonstante $Q R$-Funktion $f$ (bis auf Nullmenge) alle Werte $w \in \bar{C} N$-mal an, wo $N=\operatorname{Max}_{w \in C} n(f, w, F)$ ist. Wir können dieses Resultat noch präzisieren, indem wir für einen gegebenen Wert $w$ die Summe $n(f, w, F)+n(f, w, 9)$ betrachten. Wir beweisen zuerst den

Hilfssatz 3.1. Es sei $f$ eine nichtkonstante $Q R-F u n k t i o n, w_{0}$ ein beliebiger Punkt von $\bar{C}$ und $U\left(w_{0}, r\right)$ eine Kreisumgebung von $w_{0}$. Ist $Q$ eine Komponente von $f^{-1}\left(U\left(w_{0}, r\right)\right)$, deren Rand kompakt ist, so nimmt $f$ den Wert $w_{0}$ wenigstens einmal in $Q \cup 9(Q)$ an.

Beweis. Nach dem Hilfssatz 2.6 überdeckt die Komponente $Q$ (bis auf Nullmenge) alle Punkte von $U\left(w_{0}, r\right)$. Es sei $\left\{w_{n}\right\}, n=1,2, \ldots$ eine Punktfolge in $U\left(w_{0}, r\right)$ so dass $n\left(f, w_{n}, Q\right)>0$ für alle $n$ und $\lim w_{n}=w_{0}$. Weil die Menge $\bar{Q} \cup \mathcal{G}(Q)$ in der Topologie von $M$ kompakt $n \rightarrow \infty$ ist, hat die Punktmenge $f^{-1}\left(\left\{w_{n}\right\}\right)$ wenigstens einen Häufungspunkt $z_{0}$, der entweder in $\bar{Q}$ oder in $g(Q)$ liegt. Wegen der Stetigkeit von $f$ in $M$ (Hilfssatz 2.5) ist $f\left(z_{0}\right)=w_{0}$. Gehört $z_{0}$ zu $\bar{Q}$, so liegt es in $Q$, denn auf $\partial Q$ ist $f(z) \neq w_{0}$. Dieser Fall kommt natürlich jmmer dann vor, wenn $\bar{Q}$ kompakt ist.

Wir beweisen nun den

Satz 3.4. Ist $N=\operatorname{Max} n(f, w, F)$ für eine nichtkonstante $Q R$-Funk$w \in \bar{C}$

tion $f$, so gilt für alle $w \in \bar{C}$ die Gleichung

$$
n(f, w, M)=n(f, w, F)+n(f, w, 9)=N,
$$

wo die w-Stellen von $f$ sowohl auf $F$ als auf $g$ ihrer Multiplizität nach berechnet worden sind.

Beweis. Es sei $w_{0}$ ein beliebiger Punkt von $\bar{C}$ und $U\left(w_{0}, r\right)$ eine Kreisumgebung von $w_{0}$, so dass der Rand von $f^{-1}\left(U\left(w_{0}, r\right)\right)$ kompakt ist. Nach dem Hilfssatz 3.1 hat $f$ wenigstens eine $w_{0}$-Stelle in $Q^{i} \cup 9\left(Q^{i}\right)$ für jede Komponente $Q^{i}(i=1, \ldots, n \leqq N)$ von $f^{-1}\left(U\left(w_{0}, r\right)\right)$. Wir wählen die Zahl $r$, so dass die folgenden Bedingungen erfüllt sind: 1) Für jedes $i$ gibt es nur eine $w_{0}$-Stelle in $\left.Q^{i} \cup g_{(}{ }^{i}\right)$. 2) Für die kompakten Komponenten $Q^{i}(i=1, \ldots, h)$ gilt $n\left(f, w, \bigcup_{i=1}^{h} Q^{i}\right)=n\left(f, w_{0}, F\right) \quad$ für alle $w \in U\left(w_{0}, r\right)$. 3) Für die nichtkompakten Komponenten $Q^{j}(j=$ $h+1, \ldots, h+k)$ gilt $n\left(f, w, \bigcup_{j=h+1}^{h+k} Q^{j}\right)=n\left(f, w_{0}, 9\right)$ für alle $w \in U\left(w_{0}, r\right)$ bis auf Nullmenge $E$ (Vgl. Nr. 7 und Hilfssatz 2.7). Es sei nun $w_{1}$ ein Punkt von $U\left(w_{0}, r\right)-E$ so dass $n\left(f, w_{1}, F\right)=N$. Dann ist

$$
n\left(f, w_{1}, \bigcup_{i=1}^{h} Q^{i}\right)=n\left(f, w_{0}, F\right), n\left(f, w_{1}, \bigcup_{j=h+1}^{h+k} Q^{j}\right)=n\left(f, w_{0}, g\right)
$$


und

$$
n\left(f, w_{1}, F\right)=n\left(f, w_{1}, \bigcup_{i=1}^{h} Q^{i}\right)+n\left(f, w_{1}, \bigcup_{j=h+1}^{h+k} Q^{j}\right)=N,
$$

woraus folgt, dass

$$
n\left(f, w_{0}, F\right)+n\left(f, w_{0}, 9\right)=N .
$$

Aus dem Satz 3.4 folgt unmittelbar das folgende

Korollar 3.1. Ist $w$ ein Wert, so dass $n(f, w, F)<N$, so gibt es wenigstens eine Randkomponente $R$, so dass $f(R)=w$. Ist umgekehrt $w$ ein Wert, so dass $f(R)=w$ für eine Randkomponente $R$, so ist $n(f, w, F)<N$. Die Kapazität der Projektionsmenge $f(9)$ des idealen Randes ist gleich Null.

Die zwei ersten Behauptungen folgen aus der Gleichung (3.1). Weil für alle Werte $w \in f(g)$ die Grösse $n(f, w, F)$ kleiner als $N$ ist, folgt die letzte Behauptung aus dem Satz 3.1.

12. Aus dem Hilssatz 2.5 und aus der Definition der $Q R$-Funktion folgt der

Satz 3.5. Jede quasirationale Funktion (die in alle Randkomponenten fortgesetzt ist), ist in $M=F \cup 9$ (in der Topologie von $M$ bzw. von $\bar{C}$ ) stetig.

Weil ferner die Summe, die Differenz, das Produkt und der Quotient zweier $Q R$-Funktionen nach den bekannten Sätzen der Theorie der Grenzwerte und nach dem Hilfssatz 2.4 in jeder Randkomponente einen Grenzwert besitzen, haben wir den

Satz 3.6. Die quasirationalen Funktionen bilden einen Körper.

13. Es seien $f$ und $g$ zwei (nichtkonstante) $Q R$-Funktionen, so dass $g$ beschränkt ist ausserhalb einer kompakten Menge $K$ mit $f(K) \cap f(9)$ $=\varnothing$, und $N=\operatorname{Max}_{w \in \bar{c}} n(f, w, F)$. Es seien ferner $w \notin f(9)$ und $z_{1}(w), \ldots$, $z_{N}(w)$ die $w$-Stellen von $f$. Dann sind die symmetrischen Funktionen

$$
\begin{aligned}
& R_{1}(w)=-\sum_{k=1}^{N} g\left(z_{k}(w)\right), \\
& R_{2}(w)=\sum_{i<k} g\left(z_{i}(w)\right) g\left(z_{k}(w)\right), \\
& R_{N}(w)=(-1)^{N} g\left(z_{1}(w)\right) \ldots g\left(z_{N}(w)\right)
\end{aligned}
$$

in $\bar{C}-(f(K) \cup f(9))$ analytisch und beschränkt. Weil $f(9)$ eine Nullmenge ist, sind sie in $f(9)$ analytisch. Weil sie ferner in $f(K)$ bis auf Pole analytisch sind, sind sie rationale Funktionen von $w$. 
Jeder Wert $g\left(z_{k}(w)\right)$ genügt somit einer algebraischen Gleichung

$$
g^{N}+R_{1}(w) g^{N-1}+\ldots+R_{N}(w)=0,
$$

wo die Koeffizienten rationale Funktionen sind. Weil alle $Q R$-Funktionen in der Form

$$
a+\frac{1}{g}
$$

darstellbar sind, wo $g$ ausserhalb einer kompakten Menge beschränkt ist, gilt das obige Resultat für alle $Q R$-Funktionen.

Durch Übergang zur Fläche $F$ erhalten wir den

Satz 3.7. Sind $f$ ( $\neq$ Konstante) und $g$ zwei quasirationale Funktionen, so ist

$$
g=A \circ f
$$

wo $A$ algebraisch ist.

\section{4. Über meromorphe Differentiale}

14. Neben den $Q R$-Funktionen betrachten wir eine Klasse von meromorphen Differentialen, die mit den $Q R$-Funktionen im engen Zusammenhang stehen. Unsere Absicht ist es, eine Klasse $\Phi$ von auf einer parabolischen Riemannschen Fläche $F$ meromorphen Differentialen zu definieren, die die folgenden Eigenschaften besitzt:

$1^{\circ} \Phi$ ist ein linearer Raum.

$2^{\circ}$ Ist $f Q R$ und gehört $\varphi d z$ zu $\Phi$, so gehört auch $f \varphi d z$ zu $\Phi$.

$3^{\circ}$ Aus

$$
\int_{F-K} \int_{K}|\alpha(z)|^{2} d \sigma<\infty(K \text { kompakt })
$$

folgt $\alpha d z \in \Phi$.

$4^{\circ}$ Ein Differential $\varphi d z \in \Phi$ ist exakt: $\varphi d z=d F$ und $F$ meromorph, dann und nur dann, wenn $F$ eine $Q R$-Funktion ist.

Betreffend die Klasse $\Phi$ gilt der

Satz 4.1. Die Menge

$$
\Phi=\{\varphi d z \mid \varphi=f \alpha\}
$$

wo $f$ quasirational ist und a die Eigenschaft (4.1) besitzt, erfüllt die Bedingungen $1^{\circ}-4^{\circ}$. Sie ist die engste von allen Mengen mit den Eigenschaften $1^{\circ}-4^{\circ}$. 
Beweis. Die letzte Behauptung folgt daraus, dass jede Menge, die $1^{\circ}-4^{\circ}$ erfüllt, für jedes $\alpha$ mit der Eigenschaft (4.1) und für jede $Q R$-Funktion $f$ das Differential $f \alpha d z$ enthält.

Beweis von $1^{\circ}$.

a) $\varphi d z \in \Phi$ impliziert trivialerweise $C \varphi d z \in \Phi$ (C Konstante).

b) $\varphi_{1} d z, \varphi_{2} d z \in \Phi$ impliziert $\left(\varphi_{1}+\varphi_{2}\right) d z \in \Phi$. Beweis: Erstens ein Spezialfall: Es seien $\varphi_{1}=f \alpha, \varphi_{2}=\beta$ und $K$ eine kompakte Menge, so dass $\alpha, \beta$ die Bedingung (4.1) erfüllen und $f$ die Form

$$
f=a+\frac{1}{f_{1}}
$$

hat, wo $f_{1}$ ausserhalb $K$ beschränkt ist. Hieraus folgt

$$
\begin{gathered}
\left(\varphi_{1}+\varphi_{2}\right) d z=(f \alpha+\beta) d z=\left[\left(a+\frac{1}{f_{1}}\right) \alpha+\beta\right] d z \\
=\frac{1}{f_{1}}\left[a+f_{1}(a \alpha+\beta)\right] d z \in \Phi,
\end{gathered}
$$

weil die Grösse $\alpha+f_{1}(\alpha \alpha+\beta)$ der Bedingung (4.1) genügt und $\frac{1}{f_{1}} Q R$ ist.

Allgemeiner Fall: Es seien $f, g \quad Q R$ und $\alpha, \beta$ wie oben. Dann ist

$$
\varphi_{1}+\varphi_{2}=f \alpha+g \beta=f\left(\alpha+\frac{g}{f} \beta\right)=f \varphi,
$$

wo nach dem obigen Spezialfall $\varphi d z=\left(\alpha+\frac{g}{f} \beta\right) d z \in \Phi$ und somit $\varphi=f_{1} \alpha_{1}$ ist, wo $f_{1} Q R$ ist und $\alpha_{1}$ der Bedingung (4.1) genügt. Dann ist aber

$$
\left(\varphi_{1}+\varphi_{2}\right) d z=f\left(f_{1} \alpha_{1}\right) d z=\left(f f_{1}\right) \alpha_{1} d z \in \Phi,
$$

und die Behauptung ist bewiesen.

Beweis von $2^{\circ}$. Sind $f, f_{1} Q R$ und genügt $\alpha$ der Bedingung (4.1), so ist

$$
f_{1}(f x) d z=\left(f_{1} f\right) \alpha d z \in \Phi .
$$

Beweis von $3^{\circ}$. Die Differentiale $\alpha d z$, wo $\alpha$ der Bedingung (4.1) genügt, bilden einen linearen Unterraum von $\Phi$ mit $f \equiv 1$.

Beweis von $4^{\circ}$. a) Für jede $Q R$-Funktion $f$ gilt $d f \in \Phi$. Bewejs: Erstens ein Spezialfall: Ist $f$ beschränkt ausserhalb einer kompakten Menge $K$, so gilt

$$
\int_{F-K}\left|\frac{d f}{d z}\right|^{2} d \sigma<\infty
$$

und $d f \in \Phi$ nach der Bedingung $3^{\circ}$. 
Allgemeiner Fall: Es sei $f=a+\frac{1}{f_{1}}$, wo $f_{1}$ ausserhalb $K$ beschränkt ist. Nach dem obigen Spezialfall gilt $d f_{1} \in \Phi$ und ferner

$$
d f=-\frac{1}{f_{1}^{2}} d f_{1} \in \Phi,
$$

denn die Funktion $-\frac{1}{f_{1}^{2}}$ ist $Q R$.

b) Ist $F$ meromorph und gehört $d F$ zu $\Phi$ (d.h. $d F=f \alpha d z, f$ ist $Q R$ und $\alpha$ genügt (4.1)), so ist $F Q R$.

Beweis von b). Es soll bewiesen werden, dass $F$ in jeder Randkomponente $R$ einen (endlichen oder unendlichen) Grenzwert besitzt. Fall I: Es gibt eine Umgebung $Q$ von $R$, so dass

$$
\iint_{Q}\left|F^{\prime}(z)\right|^{2} d \sigma<\infty
$$

Dann ist $F$ in $Q$ beschränkt und hat in $R$ einen endlichen Grenzwert. Fall II: Es ist

$$
\iint_{Q}\left|F^{\prime}(z)\right|^{2} d \sigma=\infty
$$

für alle Umgebungen $Q$ von $R$, woraus folgt, dass $\lim _{z \rightarrow R} f(z)=\infty$ ist. Es sei $Q$ eine Umgebung von $R$, so dass 1) der Rand von $Q$ kompakt ist und aus einer endlichen Menge von geschlossenen Kurven $\Gamma(1)$ besteht, auf welchen $|f(z)|=1$ gilt (diese Normierung ist immer möglich, wenn man statt $f$ die Funktion $C f$ betrachtet); 2) $D_{Q}(\alpha)=\iint_{Q}|\alpha(z)|^{2} d \sigma<\infty$; 3) $f(z) \neq 0, \infty$ in allen Punkten von $Q \cup 9(Q)$, vom Punkte $R$ abgesehen. Wir bezeichnen $f(z)=r e^{i \varphi}, F(z)=U+i V$ und

$$
\Gamma(t)=\{z \in Q|| f(z) \mid=t\}, Q(t)=\{z \in Q|| f(z) \mid<t\} .
$$

Die Gebiete $Q(t)$ (die im allgemeinen nichtkompakt sind) bilden eine Ausschöpfung von $Q$, wenn $t \rightarrow \infty$. Die Kurve $\Gamma(t)$ ist kompakt, wenn $t$ zu einer offenen Teilmenge $A$ des Intervalles $(1, \infty)$ gehört, deren Komplement das Lebesguesche Mass Null hat. Ist $t$ ein Punkt von $A$, bilden wir ein Teilgebiet $Q(t, \gamma)$ von $Q(t)$, dessen Rand aus $\Gamma(1), \Gamma(t)$ und aus einer endlichen Menge $\gamma$ von anderen geschlossenen analytischen Kurven besteht, so dass die Menge $\overline{Q(t, \gamma)}$ kompakt ist. Die Funktion $\omega$ sei in $Q(t, \gamma)$ harmonisch mit den Randwerten 0 auf $\Gamma(1)$ und $\log t$ auf $\Gamma(t)$ und $\gamma$. Wir bilden das Integral 


$$
I(t, \gamma, \lambda)=\int_{\omega=\lambda} U d V=\int_{\omega=\lambda} U \frac{d U}{d \lambda} d \bar{\omega}=\frac{1}{2} \frac{d}{d \lambda} \int_{\omega=\lambda} U^{2} d \bar{\omega}
$$

wo $\bar{\omega}$ die konjugierte Funktion von $\omega$ ist und $0 \leqq \lambda \leqq \log t$. Andererseits gilt, wenn man

$$
Q(t, \gamma, \lambda)=\{z \in Q(t, \gamma) \mid \omega(z)<\lambda\}
$$

bezeichnet,

$$
\iint_{Q(t, \lambda, \gamma)}\left|F^{\prime}(z)\right|^{2} d \sigma=I(t, \gamma, \lambda)-I(t, \gamma, 0)
$$

woraus

$$
\begin{aligned}
\frac{1}{2} \frac{d}{d \lambda} \int_{\omega=\lambda} U^{2} d \bar{\omega} & =I(t, \gamma, 0)+\int_{Q(t, \gamma, \lambda)}\left|F^{\prime}(z)\right|^{2} d \sigma \\
& =I(t, \gamma, 0)+\iint_{Q(t, \gamma, \lambda)}|f|^{2}|\alpha|^{2} d \sigma \\
& \leqq I(t, \gamma, 0)+t^{2} D_{Q}(\alpha)
\end{aligned}
$$

folgt. Durch Integration von $\lambda=0$ bis $\lambda=\log t$ ergibt sich hieraus

$$
\frac{1}{2} \int_{\Gamma(t) \cup \gamma} U^{2} d \bar{\omega}-\frac{1}{2} \int_{\Gamma(1)} U^{2} d \bar{\omega} \leqq\left[I(t, \gamma, 0)+t^{2} D_{Q}(\alpha)\right] \log t .
$$

Wir lassen nun die Kurvenmenge $\gamma$ gegen $g(Q)$ konvergieren. Weil die Funktion $U$ in $Q$ beschränkt ist und die Fläche $F$ parabolisch ist, konvergiert das Integral $\int_{\gamma} U^{2} d \bar{\omega}$ gegen Null. Die Funktion $\omega$ konvergiert gegen die Funktion $\log |f(z)|$, und die Ungleichung (4.2) geht, weil die Kurven $\Gamma(1)$ und $\Gamma(t)$ analytisch sind, in die Ungleichung

$$
\begin{aligned}
\int_{\Gamma(t)} U^{2} d \varphi & \leqq \int_{\Gamma(1)} U^{2} d \varphi+2\left[\int_{\Gamma(1)} U d V+t^{2} D_{Q}(\alpha)\right] \log t \\
& =a^{\prime}+b^{\prime} \log t+c^{\prime} t^{2} \log t
\end{aligned}
$$

über, wo $a^{\prime}, b^{\prime}, c^{\prime}$ Konstanten sind. Auf dieselbe Weise erhält man die Ungleichung

$$
\int_{\Gamma(t)} V^{2} d \varphi \leqq a^{\prime \prime}+b^{\prime \prime} \log t+c^{\prime \prime} t^{2} \log t
$$

und durch Addition die Ungleichung 


$$
\int_{\Gamma(t)}|F(z)|^{2} d \varphi=\int_{\Gamma(t)}\left(U^{2}+V^{2}\right) d \varphi \leqq a+b \log t+c t^{2} \log t,
$$

wo $a, b, c$ Konstanten sind. Hieraus ergibt sich ferner, wenn $t$ grösser als eine gewisse Zahl $t_{0}$ ist,

$$
\log ^{+} \int_{\Gamma^{\prime}(t)}|F(z)|^{2} d \varphi \leqq A^{\prime} \log t \quad\left(A^{\prime} \text { Konstante }\right),
$$

wo $\log ^{+} a$ die Grösse $\operatorname{Max}(\log a, 0)$ bedeutet. Andererseits gilt nach dem Satz des arithmetisch-geometrischen Mittels die Ungleichung

$$
\frac{\int_{\Gamma(t)} \log ^{+}|F|^{2} d \varphi}{\int_{\Gamma(t)} d \varphi} \leqq \log +\frac{\int_{\Gamma(t)}|F|^{2} d \varphi}{\int_{\Gamma(t)} d \varphi}+\log 2,
$$

woraus (wenn $t$ grösser als eine gewisse Zahl $t_{1} \geqq t_{0}$ ist)

$$
\int_{\Gamma(t)} \log ^{+}|F| d \varphi \leqq A^{\prime \prime} \log t \quad\left(A^{\prime \prime} \text { Konstante }\right)
$$

folgt, denn das Integral $\int_{\Gamma(t)} d \varphi=\int_{\Gamma^{(}(t)} d \arg f$ ist beschränkt.

Es sei nun $w$ eine beliebige komplexe Zahl und das Gebiet $Q(t, \gamma, \lambda)$ und die Funktionen $\omega, \bar{\omega}$ wie oben. Aus dem Argumentenprinzip fclgt

$$
\begin{gathered}
\frac{d}{d \lambda} \int_{\omega=\lambda} \log |F-w| d \bar{\omega}=\int_{\omega=\lambda} \frac{d}{d \lambda} \log |F-w| d \bar{\omega}=-\int_{\omega=\lambda} d \arg (F-w) \\
=2 \pi n(F, w, Q(t, \gamma, \lambda))-\int_{\Gamma(1)} d \arg (F-w)
\end{gathered}
$$

und hieraus durch Integration von $\lambda=0$ bis $\lambda=\log t$

$$
\begin{gathered}
\int_{\Gamma(t) \cup_{\gamma}} \log |F-w| d \bar{\omega}-\int_{\Gamma(1)} \log |F-w| d \bar{\omega} \\
=2 \pi \int_{0}^{\log t} n(F, w, Q(t, \gamma, \lambda)) d \lambda-\log t \cdot \int_{\Gamma(1)} d \arg (F-w),
\end{gathered}
$$

woraus 


$$
2 \pi \int_{0}^{\log t} n(F, w, Q(t, \gamma, \lambda)) d \lambda
$$

$$
\leqq \int_{\Gamma(t) \cup_{\gamma}} \log ^{+}|F-w| d \bar{\omega}-\int_{\Gamma(1)} \log |F-w| d \bar{\omega}+\log t \cdot \int_{\Gamma(1)} d \arg (F-w)
$$

folgt. Weil das erste Integral in der Form

$$
\int_{0}^{\log t} n(F, w, Q(t, \gamma, \lambda)) d \lambda=n(F, w, Q(t, \gamma)) \log t-\sum_{F\left(z_{n}\right)=w} \omega\left(z_{n}\right)
$$

geschrieben werden kann, sieht man leicht ein, dass es für $\gamma \rightarrow g(Q)$ gegen das Integral

$$
\int_{0}^{\log t} n(F, w, Q(r)) d \log r
$$

konvergiert. Das Integral $\int_{\gamma} \log ^{+}|F-w| d \bar{\omega}$ dagegen konvergiert gegen Null. Aus (4.4) folgt dann

$$
\begin{gathered}
2 \pi \int_{0}^{\log t} n(F, w, Q(r)) d \log r \\
\leqq \int_{\Gamma(t)} \log ^{+}|F-w| d \varphi-\int_{\Gamma(1)} \log |F-w| d \varphi+\log t \cdot \int_{\Gamma(1)} d \arg (F-w)
\end{gathered}
$$

und wegen (4.3) und der Ungleichung

$$
\log ^{+}|F-w| \leqq \log ^{+}\left|F^{\prime}\right|+\log ^{+}|w|+\log 2,
$$

wenn $t$ grösser als eine gewisse Zahl $t_{2} \geqq t_{1}$ ist,

$$
\int_{0}^{\log t} n(F, w, Q(r)) d \log r \leqq A \log t \quad(A \text { Konstante }) .
$$

Diese Ungleichung, die für alle $t \geqq t_{2}$ gültig ist, für welche die Kurve $\Gamma(t)$ kompakt ist, zeigt, dass die Grösse $n(F, w, Q(r))$ für $r \rightarrow \infty$ beschränkt bleibt. Die Funktion $F$ nimmt somit jeden komplexen Wert in $Q$ nur in einer endlichen Menge an, woraus folgt, dass $F$ in $R$ einen (endlichen oder unendlichen) Grenzwert besitzt. 


\section{Unterklassen von meromorphen Funktionen und Differentialen}

16. Wir betrachten noch eine Teilmenge von $Q R$-Funktionen und eine Teilmenge von $\Phi$.

Es sei $f$ eine meromorphe Funktion, die ausserhalb einer kompakten Menge $K$ der parabolischen Riemannschen Fläche $F$ den Ungleichungen

$$
0<m \leqq \mid f(z) \leqq M<\infty
$$

genügt und somit $Q R$ ist. Dann ist die Funktion $u=\log |f|$ auf $F$ bis auf endlich viele logarithmische Singularitäten harmonisch und genügt den Ungleichungen

$$
\log m \leqq u \leqq \log M
$$

woraus folgt, dass

$$
\iint_{F-K}|\operatorname{grad} u|^{2} d \sigma=\int_{F-K} \int_{K}\left|\frac{d \log f}{d z}\right|^{2} d \sigma<\infty .
$$

Hilfssatz 5.1. Das Differential $x d z=d \log f$ hat die folgenden Eigenschaften:

$$
\int_{F-K} \int_{K}|\alpha(z)|^{2} d \sigma<\infty(\text { K kompakt }) .
$$

$2^{\circ}$ Die Perioden $\int_{\gamma} \alpha d z$ sind für alle Zyklen $\gamma$ rein imaginär und von der Form n2лi ( $n$ eine ganze Zahl).

$3^{\circ}$ Wenn der Zyklus d die Fläche zerlegt: $F=F_{1} \cup F_{2} \cup d$, so dass $K \subset F_{1}$, so ist

$$
\int_{d} \alpha d z=0
$$

$4^{\circ}$ Ist $z=z_{0}$ ein k-facher Pol (bzw. eine k-fache Nullstelle) von $f$, so besitzt $\alpha$ in $z_{0}$ einen einfachen Pol mit dem Residuum $k$ (bzw. $-k$ ).

$5^{\circ}$ Es gilt

$$
\int_{\gamma} \alpha d z=0
$$

für jeaen Zyklus $\gamma$, der ausserhalb einer gewissen kompakten Menge $K$ liegt. Beweis. $1^{\circ}$ Folgt aus (5.2).

$2^{\circ}$ Folgt aus $\int_{\gamma} \alpha d z=\int_{\gamma} d \arg f$. 
$3^{\circ}$ Folgt daraus, dass $\iint_{F_{2}}|\alpha|^{2} d \sigma<\infty$ und $F$ parabolisch ist.

$4^{\circ}$ Beweis evident.

$5^{\circ}$ Es sei $l$ eine Halbgerade in der $w$-Ebene von $w=0$ bis $w=\infty$, so dass $l \cap f(9)=\varnothing$. Dann hat die kompakte Menge $K=f^{-1}(l)$ die behauptete Eigenschaft. Es sei nämlich $\gamma$ ein Zyklus, der in $F-K$ liegt, und $f(\gamma)$ seine Projektionskurve. Dann gilt

$$
\int_{\gamma} d \log f=\int_{f(\gamma)} d \log w=0
$$

denn $f(\gamma)$ liegt in $\bar{C}-l$.

Umgekehrt, wenn ein meromorphes Differential adz die Bedingungen $1^{\circ}$ und $2^{\circ}$ erfüllt, ist

$$
f(z)=e^{\int d z}
$$

eine meromorphe Funktion, die der Bedingung (5.1) genügt. Das Differential $\alpha d z$ hat auch die Eigenschaften $3^{\circ}-5^{\circ}$.

17. Wir beweisen noch einen Satz betreffend den Zusammenhang zwischen den oben behandelten Klassen von meromorphen Funktionen bzw. Differentialen.

Satz 5.1. $1^{\circ}$ Die meromorphen Funktionen, die ausserhalb einer kompakten Menge einer parabolischen Riemannschen Fläche F der Ungleichung (5.1) genügen, bilden eine multiplikative Untergruppe $G$ von QR-Funktionen.

$2^{\circ}$ Die meromorphen Differentiale mit den Eigenschaften $1^{\circ}$ und $2^{\circ}$ (Hilfssatz 5.1) bilden einen linearen Unterraum $\Phi_{0}$ von $\Phi$ mit ganzzahligen (reellen) Multiplikatoren.

$3^{\circ}$ Aus $f \in G$ folgt $\alpha d z=d \log f \in \Phi_{0}$, und umgekehrt: Aus $\alpha d z \in \Phi_{0}$ folgt $f=e^{\int \alpha d x} \in G$.

$4^{\circ}$ Die Menge der Funktionen von der Form

$$
\frac{A f+B}{C f+D}, f \in G, A, B, C, D \text { komplexe Zahlen },|C|^{2}+|D|^{2}>0
$$

ist identisch mit der Menge der QR-Funktionen.

Beweis. Die Behauptungen $1^{\circ}-3^{\circ}$ sind evident.

$4^{\circ}$ Es sei $f$ eine $Q R$-Funktion und $a, b$ zwei komplexe Zahlen, die nicht zu $f(9)$ gehören. Dann gibt es eine kompakte Menge $K$ von $F$ und die positiven reellen Zahlen $m$ und $M$, so dass in $F-K$ 


$$
m \leqq\left|\frac{f(z)-a}{f(z)-b}\right| \leqq M
$$

gilt. Dann ist aber $f_{1}=\frac{f-a}{f-b}$ eine Funktion der Gruppe $G$, und

$$
f=\frac{b f_{1}-a}{f_{1}-1}
$$

ist von der Form (5.3). Umgekehrt, jede Funktion von der Form (5.3) ist quasirational.

\section{Literatur}

[1] Ahlfors, L.: The method of orthogonal decomposition for differentials on open Riemann surfaces. - Ann. Acad. Sci. Fenn. A I 249/7 (1958).

[2] - - and Sario, L.: Riemann surfaces. - Princeton University Press (1960).

[3] Herns, M.: Riemann surfaces of infinite genus. - Ann. of Math. 55:2 (1952).

[4-6] Myrberg, L.: Über meromorphe Funktionen auf endlich vielblättrigen Riemannschen Flächen. - Ann. Acad. Sci. Fenn. A 1286 (1960); II Ibid. 301 (1961); III ibid. 311 (1961).

[7] -»- Über meromorphe Funktionen auf nullberandeten Riemannschen Flächen. - Ann. Acad. Sci. Fenn. A I 312 (1962).

[8] Nevanlinna, R.: Uniformisierung. - Springer (1953).

[9] Ozawa, M.: Meromorphic functions on certain Riemann surfaces. - Proc. Amer. Math. Soc. 16 (1965).

[10] Royden, H. L.: Riemann surfaces with the AB-maximum principle. - Ann. Acad. Sci. Fenn. A I $336 / 16$ (1963).

[11] - - Riemann surfaces with the absolute AB-maximum principle. - Proc. of the conference on complex analysis, Minneapolis 1964, Springer (1965).

[12] TsuJ, M.: On a Riemann surface, which is conformally equivalent to a Riemann surface with a finite spherical area. - Comment. Math. Univ. Sancti Pauli VI 1 (1957). 